\title{
Modelling of droplet heating and evaporation
}

\author{
Sergei S Sazhin
}

\section{Introduction}

The modelling of droplet heating and evaporation has been extensively studied since the beginning of the last century, and the results have been summarised in numerous reviews and monographs including $[1,2,3]$. These studies have been mainly motivated by engineering, environmental and pharmaceutical applications of the results of this modelling. These processes are an integral part of the processes leading to autoignition of the automotive fuel vapour/air mixture in Diesel engines [4]. The following analysis will concentrate primarily on the modelling of automotive fuel droplets, although most of the results may have a much wider range of application. There will be some overlap with the results presented in $[1,2,3]$ and the analysis of the most recent publications, not included in [1, 2, 3], will be reported. As in the above-mentioned publications, some topics related to droplet heating and evaporation will not be covered. These include heating and evaporation of droplets during their interaction with walls, and the Soret effect [5]. The analysis of purely experimental papers and papers focused on multi-dimensional simulations of these processes will be limited. This chapter is intended to be complementary to reviews $[6,7]$, which look mainly at the ignition and combustion of individual droplets and arrays of droplets.

The approaches to modelling of non-evaporating droplets are discussed in Section 2. The hydrodynamic models for droplet heating and evaporation of monocomponent droplets are presented in Section 3. The hydrodynamic heating and evaporation models for multi-component droplets are summarised in Section 4. Section 5 considers the kinetic and molecular dynamics models.

Sir Harry Ricardo Laboratories, Advanced Engineering Centre, School of Computing, Engineering and Mathematics, University of Brighton, Brighton, BN2 4GJ, UK, e-mail: S.Sazhin@brighton.ac.uk 


\section{Heating of non-evaporating droplets}

The most widely used model for convective droplet heating is the one based on the assumption that liquid thermal conductivity is infinitely large. In this model the evolution of droplet temperature with time is inferred from the energy balance equation. This approach is almost universally used in research and commercial Computational Fluid Dynamics (CFD) codes and many original investigations of the problem of droplet heating, including the most recent ones $[8,9,10,11]$.

The simplest way to take into account the effects of temperature gradient within droplets was based on the solution to the one-dimensional (1D) heat transfer equation, assuming that the heating process is spherically symmetric. This equation was solved either numerically [12] or analytically [2]. It was shown that the analytical solution to this equation in the liquid phase with Robin boundary conditions (see Solution (10)) is particularly useful for practical applications in Computational Fluid Dynamics codes. This approach was implemented into the ANSYS Fluent CFD code using User-Defined Functions (UDF) [13].

The above-mentioned solution is strictly valid only for stationary spherical droplets, but it was generalised to the case of moving droplets based on the socalled Effective Thermal Conductivity (ETC) model [2]. In this model, the effects of droplet motion on the heat transfer processes inside the droplet were taken into account by replacing the liquid thermal conductivity $\left(k_{l}\right)$ with the effective thermal conductivity $k_{\mathrm{eff}}=\chi_{T} k_{l}$, where the values of $\chi_{T}$ varied from 1 for stationary droplets to 2.72 for fast moving droplets. This model could predict the average surface temperature of the droplets, which is particularly useful for many engineering applications. In the limiting case where liquid thermal conductivity is infinitely large, the effects of temperature gradient inside droplets and the effect of recirculation can be ignored. This model is known as the Infinite Thermal Conductivity (ITC) model.

The solution to the 1D heat transfer equation for spherical droplets shows that the dependence of temperature on the distance from the droplet centre is close to parabolic (except at the very beginning of the heating process). This allows one to assume that this dependence is parabolic and is characterised by two temperatures: at the centre and at the surface of the droplet. This model is known as the parabolic model [2]. The values of these temperatures were obtained from analysis of the energy balance equation at the surface of the droplet. The modification of this model made it applicable both at the beginning of the heating process and at times when the temperature profiles inside droplets are close to parabolic [2].

The limitations of the parabolic model and the complexity of the model based on the rigorous analytical/numerical solutions to the heat transfer equation inside droplets stimulated efforts to develop new models. These were more accurate than the parabolic model and more simple than the models based on the rigorous solutions to the heat transfer equation. One such model, known as the power law approximation, was suggested in [14] and further investigated in [15]. This model is based on the assumption that the temperature profile inside the droplet can be approximated as: 


$$
T(R)=c_{p 0}+c_{p p}\left(\frac{R}{R_{d}}\right)^{p},
$$

where $R$ is the distance from the droplet centre, $p$ is the parameter adjusted to replicate temperature profiles, $c_{p 0}$ and $c_{p p}$ are inferred from the values of the heat flux at the surface of the droplets and their average temperature. For $p=2$, Expression (1) describes the parabolic temperature profile.

To describe the transient process it was assumed that $p$ is time dependent. At the initial stage of heating the values of $p$ were in the range 10 to 100 , and then these values decreased with time, approaching 2 , with $p \geq 2$ at all times.

The model developed in [16] is also based on (1) but with $c_{p 0}=T_{c}$ and $c_{p p}=$ $T_{s}-T_{c}$, where $T_{c}$ and $T_{s}$ are temperatures at the centre and surface of the droplet, respectively. An empirical formula for $p$ was obtained based on the distribution of temperature predicted by the rigorous 1D solution. One of the limitations of this approximation is that it can predict only monotonic temperature profiles. This limitation was overcome by the so-called polynomial approximation, originally suggested in [17], and further investigated in [15]. This approximation is based on the following presentation of the temperature profile inside the droplet:

$$
T(R)=c_{p 0}+c_{p 2}\left(\frac{R}{R_{d}}\right)^{2}+c_{p p}\left(\frac{R}{R_{d}}\right)^{p},
$$

where $p>2$.

The most important limitation of the power law and polynomial approximations stems from the assumption that the temperature profile is instantly established in the whole droplet volume, when one would expect that initially only a thin layer close to the droplet surface is affected by the external heat, and that this heat gradually penetrates inside the droplet. These processes are taken into account in the heat balance integral method. The method is based on the introduction of the thermal layer of time-dependent thickness $\delta(t)[18,15]$. Inside this layer, temperature is approximated by the parabolic profile, while the temperature outside this layer is assumed equal to the initial temperature:

$$
T(t) \begin{cases}c_{h 0}+c_{h 2}\left(\frac{R-\left(R_{d}-\delta\right)}{R_{d}}\right)^{2}, & R_{d}-\delta<R \leq R_{d} \\ T_{0} & 0 \leq R \leq R_{d}-\delta \leq R .\end{cases}
$$

The droplet average temperature is found from the heat balance equation for the whole droplet; thickness $\delta$ is estimated by iterations of the following equation:

$$
\delta^{(i)}=R_{d} \sqrt{\frac{2 k_{\mathrm{eff}}\left(T_{0}-\bar{T}\right)}{q_{s} R_{d}}\left[1-\frac{1}{2} \frac{\delta^{(i-1)}}{R_{d}}+\frac{1}{10}\left(\frac{\delta^{(i-1)}}{R_{d}}\right)^{2}\right]^{-1}}
$$

where $i=1,2,3, \ldots$ is the iteration number, $q_{s}$ is the heat flux at the droplet surface, $k_{\text {eff }}$ is the droplet effective thermal conductivity. In the case when $\bar{T}>T_{0}$ we expect that $q_{s}<0$. In the limiting case when $\delta^{(i)}=\delta^{(i-1)}=R_{d}$, Equation (4) reduces to: 


$$
T_{0}=\bar{T}+\frac{3 q_{s} R_{d}}{10 k_{\mathrm{eff}}}
$$

This expression coincides with the one predicted by the parabolic model for $R=R_{d}$. It was shown that the thermal layer expands to $\delta=R_{d}$ when the Fourier number Fo $=k_{\text {eff }} t /\left(c_{l} \rho_{l} R_{d}^{2}\right)\left(c_{l}\right.$ and $\rho_{l}$ are specific liquid heat capacity and density respectively) reaches 0.1 . This method, as well as the power law and polynomial approximations, were verified in [15] based on the analytical solution to the heat transfer equation inside a droplet using the Neumann boundary condition. The limits of applicability of this solution have not been investigated. A solution to this equation based upon the Robin boundary condition [2] would have been a more rigorous approach.

The approaches to droplet heating discussed so far are based on the assumption that the heat conduction equation is linear and the heat conduction process follows the Fourier law (see $[19,20,21,22]$ for possible approaches to the solution to the non-linear problem and [23, 24, 25, 26, 27, 28, 29] for the analysis of non-Fourier models of heat transfer).

The heat supplied to the droplets from the gas phase is characterised by convection heat transfer coefficient $h$. In the case of stationary droplets $h=k_{g} / R_{d}$, where $k_{g}$ is gas thermal conductivity, $R_{d}$ is the droplet radius. In many practically important cases the convective heating of droplets is described by the Nusselt number $\mathrm{Nu}=2 h R_{d} / k_{g}$. Several correlations were suggested for the estimation of $\mathrm{Nu}$ for the moving droplets, including [2]:

$$
\mathrm{Nu}=2+\beta_{c} \operatorname{Re}^{1 / 2} \operatorname{Pr}^{1 / 3},
$$

where $\operatorname{Re}$ and $\operatorname{Pr}$ are Reynolds and Prandtl numbers based on gas properties and the relative velocity of droplets, $\beta_{c}=0.6$ (Ranz and Marshall correlation) and $\beta_{c}=$ 0.552 (Frossling correlation) (see [30] for a discussion of other similar correlations). An alternative correlation for $\mathrm{Nu}$ was suggested by Clift et al. [31]:

$$
\mathrm{Nu}=1+(1+\operatorname{RePr})^{1 / 3} \max \left[1, \operatorname{Re}^{0.077}\right]
$$

for $\mathrm{Re} \leq 400$. Correlation (7) was recommended in [32]. Correlations for $\mathrm{Nu}$, inferred from experimental studies, are discussed in [33].

The most widely used model for radiative heating of droplets is based on the assumption that they are opaque grey spheres with emissivity $\varepsilon$. This approach allows us to consider the effect of radiative heating of droplets as a surface phenomenon: radiative heat fluxes are added to the convective heat fluxes at the droplet surface. This approach is used in all CFD codes we are aware of, and even in a number of original recent studies (e.g. [34]). The main assumption of this model contradicts a simple observation that one can see the bottom of a glass filled with Diesel or gasoline fuel. One might anticipate that these fuels are at least partially transparent in the infra-red part of the spectrum. Thus one would expect that droplet radiative heating takes place not at their surface but via the absorption of thermal radiation penetrating inside the droplets. 
The rigorous approach to the calculation of absorption of external thermal radiation inside fuel droplets should be based on the solution to the Maxwell equations, with boundary conditions at the droplet's surface [2]. This solution was obtained in the well-known Mie theory. Direct application of the formulae predicted by this theory is limited by the complexity of relevant calculations. In most practical applications, however, we are interested not in the details of the distribution of thermal radiation absorption inside droplets but in the integral absorption of this radiation in the whole volume of droplets. This integral absorption is characterised by the efficiency factor of absorption $Q_{a}$ (the ratio of radiative power absorbed in a droplet to the radiative power illuminating the droplet). The results of Mie calculations of $Q_{a}$ at a certain wave length $\lambda$ for a typical Diesel fuel were approximated as:

$$
Q_{a}=\frac{4 n}{(n+1)^{2}}\left[1-\exp \left(-2 \tau_{0}\right)\right]
$$

where $n \equiv n_{\lambda} \approx 1.46$ is the index of refraction, $\tau_{0}=a_{\lambda} R_{d}$ is the optical thickness of droplets, $a_{\lambda}$ is the absorption coefficient.

Using the experimentally measured values of the index of absorption $\kappa_{\lambda}=$ $a_{\lambda} \lambda /(4 \pi)$ it was found that a reasonably good approximation of the average values of $Q_{a}\left(\bar{Q}_{a}\right)$ in the ranges $5 \mu \mathrm{m} \leq R_{d} \leq 50 \mu \mathrm{m}$ and $1000 \mathrm{~K} \leq \theta_{R} \leq 3000 \mathrm{~K}$ can be described by the following expression:

$$
\bar{Q}_{a}=a R_{d}^{b}
$$

where $a$ and $b$ are polynomials (or quadratic functions) of the radiative temperature $\theta_{R}$ (external temperature in the case of optically thin media), $R_{d}$ is in $\mu \mathrm{m}$.

Approximation (9) appears to be particularly useful for implementation into CFD and research numerical codes (e.g. [35]). It was shown that the predictions of typical droplet heating and evaporation based on (9) are very close to those based on a more complex model taking into account the difference in thermal radiation absorption in different areas inside droplets [36]. Classical Mie theory can be applied to spherical droplets only. In the case of illumination of droplets of more complex shapes, more advanced mathematical tools, including the generalised Lorenz-Mie theories [37], would be needed.

\section{Hydrodynamic models (mono-component droplet heating and evaporation)}

In a number of papers, including the most recent ones [38, 39, 40], the problem of heating and evaporation of droplets was solved based on direct numerical solution of transport equations in the vicinity of individual droplets. This approach, however, cannot be applied in CFD codes and will not be considered in this chapter.

In a series of our earlier papers, summarised in [2], the heating of monocomponent evaporating spherical droplets was described by the following analytical 
solution to the heat transfer equation inside them:

$$
\begin{gathered}
T(R, t)=\frac{R_{d}}{R} \sum_{n=1}^{\infty}\left\{q_{n} \exp \left[-\kappa_{R} \lambda_{n}^{2} t\right]-\frac{\sin \lambda_{n}}{\left\|v_{n}\right\|^{2} \lambda_{n}^{2}} \mu_{0}(0) \exp \left[-\kappa_{R} \lambda_{n}^{2} t\right]-\right. \\
\left.-\frac{\sin \lambda_{n}}{\left\|v_{n}\right\|^{2} \lambda_{n}^{2}} \int_{0}^{t} \frac{\mathrm{d} \mu_{0}(\tau)}{\mathrm{d} \tau} \exp \left[-\kappa_{R} \lambda_{n}^{2}(t-\tau)\right] \mathrm{d} \tau\right\} \sin \left[\lambda_{n}\left(\frac{R}{R_{d}}\right)\right]+T_{\text {eff }}(t),
\end{gathered}
$$

where $\lambda_{n}$ are solutions to the equation:

$$
\lambda \cos \lambda+h_{0} \sin \lambda=0
$$

$$
\begin{gathered}
\left\|v_{n}\right\|^{2}=\frac{1}{2}\left(1-\frac{\sin 2 \lambda_{n}}{2 \lambda_{n}}\right)=\frac{1}{2}\left(1+\frac{h_{0}}{h_{0}^{2}+\lambda_{n}^{2}}\right) \\
q_{n}=\frac{1}{R_{d}\left\|v_{n}\right\|^{2}} \int_{0}^{R_{d}} \tilde{T}_{0}(R) \sin \left[\lambda_{n}\left(\frac{R}{R_{d}}\right)\right] \mathrm{d} R, \quad \kappa_{R}=\frac{k_{l}}{c_{l} \rho_{l} R_{d}^{2}}, \quad \mu_{0}(t)=\frac{h T_{g}(t) R_{d}}{k_{l}},
\end{gathered}
$$

$h_{0}=\left(h R_{d} / k_{l}\right)-1, \tilde{T}_{0}(R)=R T_{d 0}(R) / R_{d}$; the solution to Equation (11) gives a set of positive eigenvalues $\lambda_{n}$ numbered in ascending order $(n=1,2, \ldots)$;

$$
T_{\text {eff }}=T_{g}+\frac{\rho_{l} L \dot{R}_{d}}{h}
$$

$L$ is the specific heat of evaporation, the value of $\dot{R}_{d}$, the derivative of the droplet radius with respect to time, describes the rate of droplet evaporation; remembering that in numerical codes Solution (10) was applied at each time step, the value of $\dot{R}_{d}$ was taken from the previous time step (it was taken as zero at the first time step).

The effect of evaporation on the Nusselt number for stationary spherical droplets can be described by the following equation (the Stefan-Fuchs model):

$$
\mathrm{Nu}=\mathrm{Nu}_{0} \frac{\ln \left(1+B_{T}\right)}{B_{T}},
$$

where $\mathrm{Nu}_{0}=2 h_{0} R_{d} k_{g}=2, h_{0}=k_{g} / R_{d}$ is the convective heat transfer coefficient for a non-evaporating sphere, $B_{T}$ is the Spalding heat transfer number

$$
B_{T}=\frac{c_{p v}\left(T_{g}-T_{s}\right)}{L\left(T_{s}\right)-\left(\left|\dot{q}_{d}\right| / \dot{m}_{d}\right)},
$$

$c_{p v}$ is the specific heat capacity of fuel vapour at constant pressure, $T_{s}$ is the droplet surface temperature, $\left|\dot{q}_{d}\right|$ is heat spent on raising droplet internal energy. The droplet evaporation rate $\dot{m}_{d} \leq 0$ for stationary droplets can be estimated as:

$$
\dot{m}_{d}=-4 \pi R_{d} D_{v} \rho_{\text {total }} \ln \left(1+B_{M}\right),
$$

where $D_{v}$ is the binary diffusion coefficient of fuel vapour in air, $\rho_{\text {total }}=\rho_{v}+\rho_{g}$ is the density of the mixture of vapour and ambient air, $B_{M}$ is the Spalding mass 
transfer number defined as

$$
B_{M}=\frac{\rho_{v s}-\rho_{v \infty}}{\rho_{g s}}
$$

where subscript ${ }_{s}$ refers to the surface of the droplet, subscript ${ }_{\infty}$ refers to ambient conditions. When deriving (15) it was assumed that $\rho_{\text {total }}$ does not depend on the distance from the surface of the droplet. This is expected to be a serious limitation of the model for strongly evaporating droplets with high surface temperatures. A model in which this assumption is relaxed was developed in [41].

Note that $\rho_{v s}$ is controlled by the droplet surface temperature. This leads to a strong link between (15) and the corresponding equation for droplet heating.

Expression (15) can be presented in a more compact form:

$$
\dot{m}_{d}=-2 \pi R_{d} D_{v} \rho_{\mathrm{total}} \mathrm{Sh} B_{M},
$$

where

$$
\mathrm{Sh} \equiv \frac{2 h_{m} R_{d}}{D_{v}}=\mathrm{Sh}_{0} \frac{\ln \left(1+B_{M}\right)}{B_{M}}=\frac{2 h_{m 0} R_{d}}{D_{v}} \frac{\ln \left(1+B_{M}\right)}{B_{M}}=2 \frac{\ln \left(1+B_{M}\right)}{B_{M}}
$$

is the Sherwood number, $h_{m}$ is the convective mass transfer coefficient, subscript 0 indicates non-evaporating droplets.

An alternative expression for $\dot{m}_{d}$ for stationary droplets was derived as [2]:

$$
\dot{m}_{d}=-\frac{4 \pi k_{m} R_{d}}{c_{p v}} \ln \left(1+B_{T}\right),
$$

where $k_{m}$ is thermal conductivity of the mixture of ambient gas and fuel vapour (in the case of weak evaporation, $k_{m} \approx k_{g}$ ), $B_{T}$ is defined by (14).

Expressions (13), (15), (18) and (19) could be generalised to the case of moving evaporating droplets using the so-called 'film theory' [32]. The key concepts of this theory are film thicknesses $\delta_{T}$ and $\delta_{M}$. Ignoring the Stefan flow, they can be estimated as [32]:

$$
\delta_{T 0}=\frac{2 R_{d}}{\mathrm{Nu}_{0}-2}, \quad \delta_{M 0}=\frac{2 R_{d}}{\mathrm{Sh}_{0}-2} .
$$

For stationary droplets $\mathrm{Nu}_{0}=\mathrm{Sh}_{0}=2$. Hence, $\delta_{T 0}=\delta_{M 0}=\infty$.

The effect of droplet motion on $\mathrm{Nu}_{0}$ for non-evaporating droplets is described by Equations (6) and (7) (subscripts ${ }_{0}$ in these equations need to be added to indicate non-evaporating droplets). Similar expressions were obtained for $\mathrm{Sh}_{0}$ [32]:

$$
\begin{gathered}
\mathrm{Sh}_{0}=2+\beta_{c} \operatorname{Re}^{1 / 2} \mathrm{Sc}^{1 / 3}, \\
\mathrm{Sh}_{0}=1+(1+\mathrm{ReSc})^{1 / 3} \max \left[1, \operatorname{Re}^{0.077}\right]
\end{gathered}
$$

for $\operatorname{Re} \leq 400$, where $\mathrm{Sc}=v_{m} / D_{v}, v_{m}$ is the kinematic viscosity of the mixture of gas and vapour. $\delta_{T 0}$ and $\delta_{M 0}$, defined by (20), can be considered as thicknesses of the thermal and diffusional boundary layers. The thickening of these layers due to the effect of the Stefan flow was described by parameters $F_{T}$ and $F_{M}$ [32]: 


$$
F_{T}=\delta_{T} / \delta_{T 0}, \quad F_{M}=\delta_{M} / \delta_{M 0} .
$$

The following correlations were suggested in the ranges $0 \leq\left(B_{T}, B_{M}\right) \leq 20$ and $1 \leq(\mathrm{Pr}, \mathrm{Sc}) \leq 3$ [32]:

$$
F_{T, M}=\left(1+B_{T, M}\right)^{0.7} \frac{\ln \left(1+B_{T, M}\right)}{B_{T, M}} .
$$

Using film theory, we would expect that an increase in the film thicknesses, described by (23) and (24), would lead to a corresponding decrease in $\mathrm{Nu}_{0}$ and $\mathrm{Sh}_{0}$. The new decreased values of $\mathrm{Nu}_{0}$ and $\mathrm{Sh}_{0}$, called 'modified' Nusselt and Sherwood numbers in [32] $\left(\mathrm{Nu}^{*}\right.$ and $\left.\mathrm{Sh}^{*}\right)$, were estimated as [32]:

$$
\mathrm{Nu}^{*}=2+\frac{\mathrm{Nu}_{0}-2}{F_{T}}, \quad \mathrm{Sh}^{*}=2+\frac{\mathrm{Sh}_{0}-2}{F_{M}} .
$$

These parameters allow us to present the expressions for $\mathrm{Nu}$ and $\mathrm{Sh}$ as:

$$
\mathrm{Nu}=\mathrm{Nu}^{*} \frac{\ln \left(1+B_{T}\right)}{B_{T}}, \quad \mathrm{Sh}=\mathrm{Sh}^{*} \frac{\ln \left(1+B_{M}\right)}{B_{M}} .
$$

The introduction of $\mathrm{Nu}^{*}$ and $\mathrm{Sh}^{*}$ allows one to present (15) and (19) as [32]:

$$
\begin{gathered}
\dot{m}_{d}=-2 \pi R_{d} D_{v} \rho_{\text {total }} \mathrm{Sh}^{*} \ln \left(1+B_{M}\right), \\
\dot{m}_{d}=-\frac{2 \pi k_{m} R_{d}}{c_{p v}} \mathrm{Nu}^{*} \ln \left(1+B_{T}\right) .
\end{gathered}
$$

Note that [32]:

$$
B_{T}=\left(1+B_{M}\right)^{\varphi}-1,
$$

where

$$
\varphi=\left(\frac{c_{p v}}{c_{p g}}\right)\left(\frac{\mathrm{Sh}^{*}}{\mathrm{Nu}^{*}}\right) \frac{1}{\mathrm{Le}},
$$

Le $=k_{m} /\left(D_{v} \rho_{\text {total }} c_{p g}\right)$ is the gas Lewis number but with thermal conductivity and density equal to those of a mixture of air and vapour. ${ }^{1}$

The evaporation process leads to the inward movement of the droplet surface (liquid/vapour interface). The effect of this movement on droplet heating was considered in a series of our papers summarised in Section 4.4 of [2].

The model based on the combination of the above-mentioned model for the gas phase and the analytical solution to the heat transfer equation for the liquid phase (Solution (10)) was extensively validated [44].

Remembering (14) and (29), one can obtain the heat rate supplied to the droplet to raise (or reduce) its temperature (internal energy) in the form:

\footnotetext{
${ }^{1}$ Note that in many papers, including the most recent ones (e.g. [42]), it is assumed that $c_{p v}=c_{p g}$ in (28) and (30) which is obviously not correct. In [43] $c_{p}=c_{p v}$ in the definition of Le but $c_{p}=c_{p g}$ in the definition of Pr, which led to ambiguity in their model.
} 


$$
\dot{q}_{d}=-\dot{m}_{d}\left[\frac{c_{p v}\left(T_{g}-T_{s}\right)}{B_{T}}-L\left(T_{s}\right)\right]=-\dot{m}_{d}\left[\frac{c_{p v}\left(T_{g}-T_{s}\right)}{\left(1+B_{M}\right)^{\varphi}-1}-L\left(T_{s}\right)\right],
$$

where $\dot{q}_{d}>0$ when the droplet is heated; for stationary droplets: $\varphi=\left(\frac{c_{p v}}{c_{p g}}\right) \frac{1}{\mathrm{Le}}$.

An alternative approach to the calculation of $\dot{q}_{d}$ could be based on the analysis of the temperature distribution inside droplets, predicted by Equation (10):

$$
\dot{q}_{d}=\left.4 \pi R_{d}^{2} k_{l} \frac{\partial T}{\partial R}\right|_{R=R_{d}-0} .
$$

Having substituted (10) into (32) we obtain:

$$
\begin{gathered}
\dot{q}_{d}=4 \pi R_{d} k_{l} \sum_{n=1}^{\infty}\left\{q_{n} \exp \left[-\kappa_{R} \lambda_{n}^{2} t\right]-\frac{\sin \lambda_{n}}{\left\|v_{n}\right\|^{2} \lambda_{n}^{2}} \mu_{0}(0) \exp \left[-\kappa_{R} \lambda_{n}^{2} t\right]-\right. \\
\left.-\frac{\sin \lambda_{n}}{\left\|v_{n}\right\|^{2} \lambda_{n}^{2}} \int_{0}^{t} \frac{\mathrm{d} \mu_{0}(\tau)}{\mathrm{d} \tau} \exp \left[-\kappa_{R} \lambda_{n}^{2}(t-\tau)\right] \mathrm{d} \tau\right\}\left[-1-h_{0}\right] \sin \lambda_{n},
\end{gathered}
$$

where all notations are the same as in Solution (10).

Once the value of $\dot{q}_{d}$ has been found, the evaporation rate can be found from Equation (19). Remembering the definition of $B_{T}$, this equation can be rewritten as:

$$
\dot{m}_{d}=-\frac{4 \pi k_{g} R_{d}}{c_{p v}} \ln \left(1+\frac{c_{p v}\left(T_{g}-T_{s}\right) \dot{m}_{d}}{L\left(T_{s}\right) \dot{m}_{d}-\dot{q}_{d}}\right) .
$$

Thus we have two approaches to modelling the heating and evaporation of stationary droplets. The first is based on Equations (15) and (31) (conventional approach originally suggested in [32], Model 1), and the second is based on Equations (33) and (34) (Model 2). Detailed comparison between the predictions of these models was performed by [45]. Although these predictions were qualitatively similar, there were noticeable quantitative differences between them. The reasons behind these differences are still unclear.

The models described so far are based on the assumption that droplets are perfect spheres. However, the shapes of most actually observed droplets in engineering and environmental applications are far from spherical (see [46, 47]). In most cases the effects of non-sphericity of droplets have been investigated assuming that droplet shapes can be approximated by prolate or oblate spheroids.

The heat conduction equation inside a spheroidal body (droplet), using the Dirichlet boundary conditions, was first solved analytically more than 135 years ago [48]. This solution, however, turned out to be too complex for most practical applications. In most cases this problem (and the related problem of mass transfer inside the body) has been investigated based on the numerical solutions to the heat transfer (and mass diffusion) equations [49, 50, 51].

The problem of heat/mass transfer inside spheroidal bodies, considered in the above-mentioned papers, is complementary to the problem of heat/mass transfer from/to an ambient fluid (gas) to/from a spheroidal body, taking into account the 
relative velocity between the gas and the body, in the general case. The latter problem has been considered in numerous papers based on the numerical solutions to momentum and heat transfer equations in the ambient fluid (gas) in the ellipsoidal coordinate system. The analysis of $[52,53,54,55,56]$ was based on the assumption that the body surface was fixed. Juncu [57] took into account changes in body temperature with time, while assuming that there is no temperature gradient inside the body (the thermal conductivity of the body was assumed infinitely high).

These approaches are equally applicable to solid bodies and droplets. In the case of droplets, however, apart from heating, the evaporation processes should also be taken into account in the general case. Grow [58] was perhaps the first to solve the problem of heat and mass transfer in the vicinity of spheroidal particles assuming that their relative velocities are equal to zero, although she considered coal chars rather than droplets. One of the main limitations of this paper is that both mass and heat transfer equations were presented in the form of Laplace equations, which implies that the effects of Stefan flow from the surface of the particles were ignored. The latter effects were taken into account in the exact solutions to the mass and heat transfer equations in the gas phase around a spheroidal droplet suggested in [59]. In that paper it was assumed that the temperatures at all points at the surface of a droplet are the same and constant, and the droplet's shape remains spheroidal. A combined problem of spheroidal droplet heating and evaporation, similar to the one studied in [59], was considered in [60]. As in [59], the authors of [60] based their analysis on the solution to the species conservation equation in the gas phase and assumed that the thermal conductivity of droplets is infinitely large. In contrast to [59], the authors of [60] took into account the relative velocities of droplets, assuming that the dependencies of the Nusselt and Sherwood numbers on the Reynolds and Prandtl numbers are the same as those for the spherical droplets. Also, they took into account the time dependence of droplet temperatures and sizes, although their analysis focused on oblate droplets only.

Strotos et al. [61] presented CFD analysis of the evaporation of nearly spherical suspended droplets. They solved the Navier-Stokes, energy conservation and species transport equations; the Volume of Fluid (VOF) approach was used to capture the liquid-gas interface.

As follows from an overview of the models described above, the general problem of heating and evaporation of spheroidal droplets is far from resolved. We believe, however, that the results presented in [59] could be considered a starting point for solving this problem at least for slightly deformed spheroids.

The model described in [59] was generalised to the case of oscillating droplets under the assumption that the process can be considered quasi-steady-state [62]. The instantaneous and average mass and heat transfer rates over an oscillation period were functions of the oscillating frequency and amplitude. The results were compared with the predictions of the approximate model described by Mashayek [63]. The model was able to capture different evaporating mechanisms for oblate and prolate droplets.

The results of the generalisation of the model described above to the case of triaxial ellipsoidal droplets are presented in [64]. In this paper, a new analytical 
model for heat and mass transfer from deformed droplets was developed, based on the solutions to the species and energy conservation equations under steady-state conditions. It was shown that the droplet deformation enhances both the total and local mass and heat transfer. The evaporation rate from deformed droplets, having the same volume and surface area, was shown to be at a maximum for the prolate droplet and at a minimum for the oblate droplet. Purely numerical investigation of fluid flow and heat transfer from heated spheroids was conducted in [65].

The solutions developed in [59] for the gas phase surrounding a spheroidal droplet were used as boundary conditions for the solutions to heat/mass equations in the liquid phase [66]. The temperature gradients inside and at the surface of the droplets, and the changes in their shape during the heating and evaporation process were taken into account. The effects of surface tension and droplet motion on droplet heating and evaporation were ignored. The results were applied to the analysis of an $\mathrm{n}$-dodecane fuel droplet in Diesel engine-like conditions.

\section{Hydrodynamic models (multi-component droplet heating and evaporation)}

All models for mono-component droplets discussed in the previous section are applicable to multi-component droplets. In addition to the processes considered in the previous section, however, for multi-component droplets we need to take into account that different components evaporate at different rates, creating concentration gradients in the liquid phase. The latter leads to the liquid phase mass diffusion of species described by the diffusion equation for the mass fractions of each component. The simplest form of this equation, when only the radial diffusion is accounted for and species diffusion coefficient $D_{l}$ is constant, can be presented as [2]:

$$
\frac{\partial Y_{l i}}{\partial t}=D_{l}\left(\frac{\partial^{2} Y_{l i}}{\partial R^{2}}+\frac{2}{R} \frac{\partial Y_{l i}}{\partial R}\right)
$$

where subscripts $l_{l}$ and ${ }_{i}$ indicate liquid phase and type of species, respectively.

This equation needs to be solved subject to the boundary condition at the surface:

$$
\left.\frac{\partial Y_{l i}}{\partial R}\right|_{R=R_{d}-0}=\frac{D_{v} \rho_{\mathrm{total}} \ln \left(1+B_{M}\right)}{D_{l} \rho_{l} R_{d}}\left(Y_{l i}-\varepsilon_{i}\right),
$$

where

$$
\varepsilon_{i}=\frac{Y_{v s i}}{\sum_{i} Y_{v s i}}
$$

(subscript ${ }_{v}$ indicates the vapour phase) and at the centre of the droplet:

$$
\left.\frac{\partial Y_{l i}}{\partial R}\right|_{R=0}=0
$$


and the relevant initial conditions. Note that Condition (38) can be replaced by a more general requirement, that $Y_{l i}(R, t)$ are twice continuously differentiable functions at $R \leq R_{d}$.

In the equilibrium state, the partial pressure of the $i$ th vapour species at the surface of the droplet can be found from the equation:

$$
p_{v i}=\gamma_{i} X_{l s i} p_{v i}^{*}
$$

where $X_{l s i}$ is the molar fraction of the $i$ th species in the liquid at the droplet surface, $p_{v i}^{*}$ is the partial vapour pressure of the $i$ th species when $X_{l i}=1, \gamma_{i}$ is the activity coefficient. If $\gamma_{i}=1$ Equation (39) leads to Raoult's law:

$$
p_{v i}=X_{l i} p_{v i}^{*}
$$

In many engineering applications, $D_{l}$ was considered to be either infinitely small (multi-component droplets were modelled as mono-component ones) or infinitely large (perfect mixing of species). Both these simplified approaches, however, can lead to unacceptably large errors in predicted droplet temperatures and droplet evaporation times compared with the prediction of the model taking into account finite species diffusion rates inside droplets. Where species diffusion was taken into account, this was mainly performed based on the numerical solution to Equation (35) (e.g. [67, 68, 69]). In contrast to this approach, in a series of our papers, the results of which are summarised in [2], a new approach to this problem based on the analytical solution to (35), subject to boundary conditions (36) and (38), was suggested. Assuming that $R_{d}=$ const, this solution for a short time step, subject to the initial condition $Y_{l i}(t=0)=Y_{l i 0}(R)$ was obtained in the form [2]:

$$
\begin{gathered}
Y_{l i}=\varepsilon_{i}+\frac{1}{R}\left\{\exp \left[D_{l}\left(\frac{\lambda_{0}}{R_{d}}\right)^{2} t\right]\left[q_{Y i 0}-Q_{Y 0} \varepsilon_{i}\right] \sinh \left(\lambda_{0} \frac{R}{R_{d}}\right)\right. \\
\left.+\sum_{n=1}^{\infty}\left[\exp \left[-D_{l}\left(\frac{\lambda_{n}}{R_{d}}\right)^{2} t\right]\left[q_{Y i n}-Q_{Y n} \varepsilon_{i}\right] \sin \left(\lambda_{n} \frac{R}{R_{d}}\right)\right]\right\},
\end{gathered}
$$

where $\lambda_{0}$ and $\lambda_{n}(n \geq 1)$ are solutions to equations

$$
\tanh \lambda=-\frac{\lambda}{h_{Y 0}} \quad \text { and } \quad \tan \lambda=-\frac{\lambda}{h_{Y 0}},
$$

respectively, $h_{Y 0}=-\left(1+\frac{\alpha_{m} R_{d}}{D_{l}}\right)$,

$$
Q_{Y n}=\left\{\begin{array}{lll}
-\frac{1}{\left\|v_{Y 0}\right\|^{2}}\left(\frac{R_{d}}{\lambda_{0}}\right)^{2}\left(1+h_{Y 0}\right) \sinh \lambda_{0} & \text { when } & n=0 \\
\frac{1}{\left\|v_{Y n}\right\|^{2}}\left(\frac{R_{d}}{\lambda_{n}}\right)^{2}\left(1+h_{Y 0}\right) \sin \lambda_{n} & \text { when } & n \geq 1
\end{array}\right.
$$




$$
\begin{gathered}
q_{Y i n}=\frac{1}{\left\|v_{Y n}\right\|^{2}} \int_{0}^{R_{d}} R Y_{l i 0}(R) v_{Y n}(R) \mathrm{d} R, \quad n \geq 0, \\
v_{Y 0}(R)=\sinh \left(\lambda_{0} \frac{R}{R_{d}}\right), \quad v_{Y n}(R)=\sin \left(\lambda_{n} \frac{R}{R_{d}}\right), n \geq 1, \\
\left\|v_{Y 0}\right\|^{2}=\int_{0}^{R_{d}} v_{Y 0}^{2}(R) \mathrm{d} R=-\frac{R_{d}}{2}\left[1+\frac{h_{Y 0}}{h_{Y 0}^{2}-\lambda_{n}^{2}}\right], \\
\left\|v_{Y n}\right\|^{2}=\int_{0}^{R_{d}} v_{Y n}^{2}(R) \mathrm{d} R=\frac{R_{d}}{2}\left[1+\frac{h_{Y 0}}{h_{Y 0}^{2}+\lambda_{n}^{2}}\right], \quad n \geq 1,
\end{gathered}
$$

$Y_{l s i}=Y_{l s i}(t)$ are liquid components' mass fractions at the droplet's surface,

$$
\alpha_{m}=\frac{\left|\dot{m}_{d}\right|}{4 \pi \rho_{l} R_{d}^{2}}=\text { const. }
$$

There are obvious typos in Equations (5.18) and (5.20) in [2] corrected in [3].

In the case of moving droplets, the distribution of mass fractions of species can be described by (41), but with $D_{l}$ replaced by the effective diffusivity $D_{\text {eff }}$ :

$$
D_{\text {eff }}=\chi_{Y} D_{l}
$$

where the coefficient $\chi_{Y}$ can be approximated as:

$$
\chi_{Y}=1.86+0.86 \tanh \left[2.225 \log _{10}\left(\operatorname{Re}_{d(l)} \mathrm{Sc}_{l} / 30\right)\right]
$$

$\mathrm{Sc}_{l}=v_{l} / D_{l}$ is the liquid Schmidt number, $v_{l}$ is the liquid kinematic viscosity. Liquid fuel transport properties and the liquid velocity just below the droplet surface were used to calculate $\operatorname{Re}_{d(l)}$. The model based on (47) and (48) is known as the Effective Diffusivity (ED) model. The model, based on the assumption that species diffusivity is infinitely fast $\left(D_{\text {eff }}=\infty\right)$ is referred to as the Infinite Diffusivity (ID) model.

As in the case of the heat transfer equation inside droplets, Solution (41) was generalised to the case of time-dependent droplet radii during the time step. Also, as in the case of mono-component droplets, the model based on (41) was validated based on the experimental results [44].

The model based on Equation (35) or its solution (41) is known as the Discrete Component Model (DCM). It is typically applicable only in the case when the number of components in the droplets is small (e.g. biodiesel droplets [70, 71]) which is not the case in most automotive fuels. An alternative approach is based on the probabilistic analysis of a large number of components (e.g. Continuous Thermodynamics approach and the Distillation Curve Model; see [2] for details). Further developments of these models led to the Quadrature Method of Moments (QMoM) [72] and Direct Quadrature Method of Moments (DQMoM). The latter method was further developed in [73] which led to the Direct Quadrature Method of Moments with delumping. In this family of models a number of additional simplifying assumptions were used, including the assumption that species inside droplets mix infinitely quickly. 
A new approach to modelling heating and evaporation of multi-component droplets, suitable for the case when a large number of components is present in the droplets, was suggested in $[74,75]$. In contrast to the previously suggested models, designed for large numbers of components, the new model takes into account the diffusion of liquid species and thermal diffusion as in the classical Discrete Component Model. This model was called the quasi-discrete model. As in the case of the Continuous Thermodynamics approach, the quasi-discrete model is based on the distribution function with respect to a particular property. Describing this property by a carbon number $n$, this function was approximated as:

$$
f_{m}(n)=C_{m}\left(n_{0}, n_{f}\right) \frac{(M(n)-\gamma)^{\alpha-1}}{\beta^{\alpha} \Gamma(\alpha)} \exp \left[-\left(\frac{M(n)-\gamma}{\beta}\right)\right],
$$

where $n_{0} \leq n \leq n_{f}$, subscripts 0 and $f_{f}$ stand for initial and final (the smallest and the largest values of $n), M$ is the molar mass, $\Gamma(\alpha)$ is the Gamma function, $\alpha$, $\beta$ and $\gamma$ are parameters that determine the shape of the distribution, the choice of $C_{m}\left(n_{0}, n_{f}\right)$ assured that $\int_{n_{0}}^{n_{f}} f_{m}(n) \mathrm{d} n=1$. Assuming that fuel includes only alkanes, $M$ (in $\mathrm{kg} / \mathrm{kmole}$ ) and $n$ can be linked by the following expression:

$$
M=14 n+2 .
$$

As follows from the previous analysis [2], the transport and thermodynamic properties of alkanes are weak functions of $n$. In this case, one can assume that their properties in a certain narrow range of $n$ are close, and replace the continuous distribution (49) with a discrete one, consisting of $N_{f}$ quasi-components with carbon numbers

$$
\bar{n}_{j}=\frac{\int_{n_{j-1}}^{n_{j}} n f_{m}(n) \mathrm{d} n}{\int_{n_{j-1}}^{n_{j}} f_{m}(n) \mathrm{d} n}
$$

and molar fractions

$$
X_{j}=\int_{n_{j-1}}^{n_{j}} f_{m}(n) \mathrm{d} n
$$

where $j$ is an integer in the range $1 \leq j \leq N_{f}$. It was assumed that all $n_{j}-n_{j-1}$ are equal, i.e. all quasi-components have the same range of values of $n$. For the case when $N_{f}=1$ the analysis of multi-component droplets is reduced to that of mono-component droplets. $\bar{n}_{j}$ are not integers in the general case and do not represent carbon numbers for actual components. Hence, this model is called the quasidiscrete model. These quasi-components, however, were treated as actual components in the conventional Discrete Component Model (DCM). This model is expected to be particularly useful when $N_{f}$ is much less than the number of actual species in the hydrocarbon mixture. All thermodynamic and transport properties of quasi-components were determined for $n=\bar{n}_{j}$. For example, partial pressures of individual quasi-components were estimated as (Raoult's law is assumed to be valid):

$$
p_{v}\left(\bar{n}_{j}\right)=X_{l s i}\left(\bar{n}_{j}\right) p^{*}\left(\bar{n}_{j}\right)
$$


where $X_{l s i}$ are the surface molar fractions of liquid quasi-components.

The main limitation of the quasi-discrete model is that it is based on the assumption that fuels consist only of alkanes, whilst the total molar fraction of alkanes (n-alkanes and iso-alkanes) is only about $40 \%$ of the overall composition of Diesel fuels (a similar conclusion could be drawn for gasoline fuel). Hence, the contribution of other components cannot be ignored. Also, even if we restrict our analysis to alkanes alone, it is not easy to approximate this distribution with a reasonably simple distribution function $f_{m}(n)$, similar to the one given by Expression (49). In [76], the quasi-discrete model was generalised to address both these problems. A realistic composition of Diesel fuels, schematically shown in Fig. 1, was used in the analysis presented in [76].

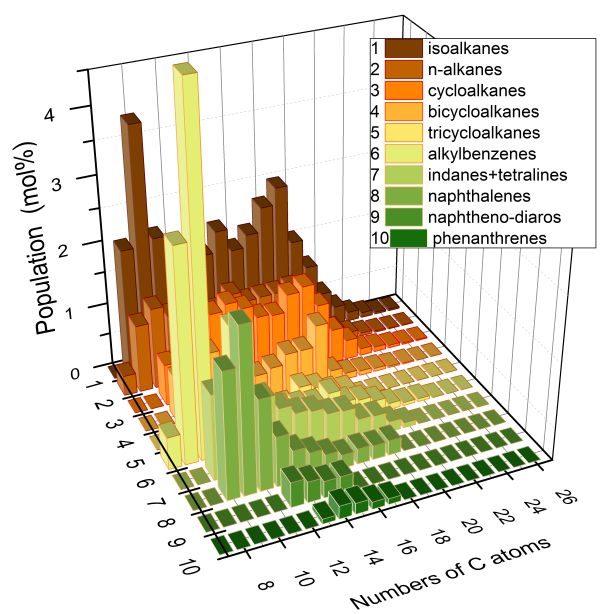

Fig. 1 Distribution functions of various hydrocarbons versus the numbers of carbon atoms in molecules in a representative sample of Diesel fuel. Reprinted from Fluid Phase Equilibria, Volume 356, Gun'ko et al., A quantum chemical study of the processes during the evaporation of real-life Diesel fuel droplets, Pages 146-156, Copyright Elsevier (2013).

The results presented in Fig. 1 were simplified, taking into account that the properties of $n$-alkanes and iso-alkanes are rather close. Observing that the contributions of tricycloalkanes, diaromatics and phenanthrenes to Diesel fuel are rather small (less than about $1.6 \%$ for each of these components) allows us to ignore the dependence of the properties of these components on the number of carbon atoms and replace these three groups with three components, tricycloalkane, diaromatic and phenanthrene, with arbitrarily chosen carbon numbers. The molar fraction of tricycloalkanes was estimated to be $1.5647 \%$, while the molar fractions of diaromatics and phenanthrenes were estimated to be $1.2240 \%$ and $0.6577 \%$, respectively. Transport and thermodynamic properties of the components are summarised in Appendices 1-7 of [76]. In the new model, the focus is shifted from the analysis of the dis- 
tribution function to the direct analysis of molar fractions of the components. These are described by the matrix $X_{n m}$, where $n$ refers to the number of carbon atoms, and $m$ refers to the groups (e.g. alkanes) or individual components (tricycloalkane, diaromatic and phenanthrene). The link between the values of $m$ and the components is shown in Table 1.

\begin{tabular}{ll}
\hline$m$ & Component \\
\hline 1 & Alkanes \\
2 & Cycloalkanes \\
3 & Bicycloalkanes \\
4 & Alkylbenzenes \\
5 & Indanes \& tetralines \\
6 & Naphthalenes \\
7 & Tricycloalkane \\
8 & Diaromatic \\
9 & Phenanthrene \\
\hline
\end{tabular}

Table 1 The relation between parameter $m$ and groups $(m=1-6)$ and components $(m=7-9)$. Reprinted from Fuel, Volume 154, Sazhin et al., A multi-dimensional quasi-discrete model for the analysis of Diesel fuel droplet heating and evaporation, Pages 238-266, Copyright Elsevier (2014).

For each $m$ the values of $\bar{n}_{j m}$ of quasi-components were introduced. As in the case of the original quasi-discrete model, $\bar{n}_{i m}$ are not integers in the general case. Due to the additional dimensions introduced by the subscript $m$, the new model is called the Multi-dimensional Quasi-discrete Model (MDQDM). The maximal number of these quasi-components/components, providing the most accurate approximation of Diesel fuel, was taken to be equal to the actual number of components (98 in the case considered in [76]). In this case, the new model reduces to the conventional Discrete Component Model (DCM). The quasi-components in the MDQDM are treated in the same way as the quasi-components in the conventional quasi-discrete model. Also, the temperature gradient and quasi-components' diffusion inside droplets are taken into account as in the quasi-discrete model.

In [76] the MDQDM was applied to the analysis of heating and evaporation of a droplet with initial radius $R_{d 0}=10 \mu \mathrm{m}$ in air with density, temperature and pressure equal to $\rho_{a}=11.9 \mathrm{~kg} / \mathrm{m}^{3}, T_{a}=880 \mathrm{~K}, p_{a}=30 \mathrm{bar}$, respectively. All transport and thermodynamic properties for Diesel fuel and its components are given in [76]. ${ }^{2}$

The plots of the droplet surface temperatures $T_{s}$ and radii $R_{d}$ versus time for a wide range of approximations of Diesel fuel are shown in Figs. 2 and 3.

As can be seen from Figs. 2 and 3, the approximation of 98 actual components by a single quasi-component leads to a noticeable under-estimation of the

\footnotetext{
${ }^{2}$ The results of most recent experimental and theoretical studies of Diesel fuel viscosity are presented in $[77,78]$
} 


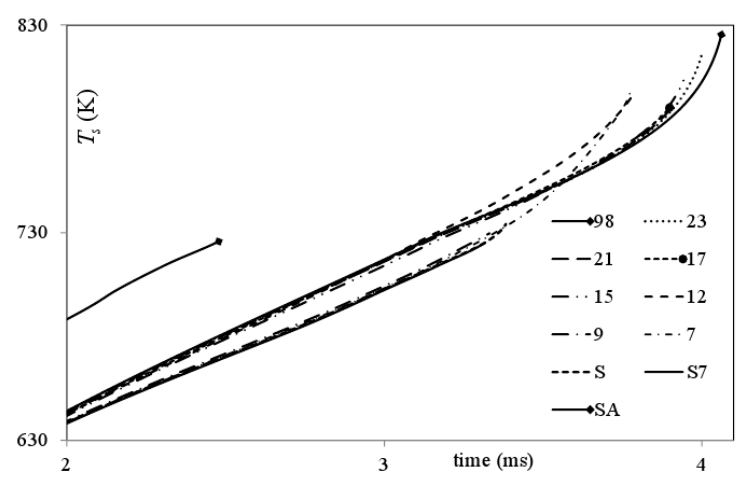

Fig. 2 The plots of the droplet surface temperatures $T_{s}$ versus time for ten approximations of Diesel fuel composition: 98 components (indicated as (98)); 23, 21, 17, 15, 12, 9 and 7 quasicomponents/components (numbers near the curves); the contributions of all groups are approximated by single quasi-components, to which the contribution of tricycloalkane is added, leading to 7 quasi-components/components (indicated as (S7)); the contribution of all 98 components is taken into account as that of a single component (indicated as (S)); the contributions of only 20 alkane components are taken into account and these are treated as a single component, with the average value of the carbon number $\left(\mathrm{C}_{14.763} \mathrm{H}_{31.526}\right.$; indicated as $(\mathrm{SA})$ ). Only the final stage of droplet heating and evaporation is shown. Reprinted from Fuel, Volume 154, Sazhin et al., A multidimensional quasi-discrete model for the analysis of Diesel fuel droplet heating and evaporation, Pages 238-266, Copyright Elsevier (2014).

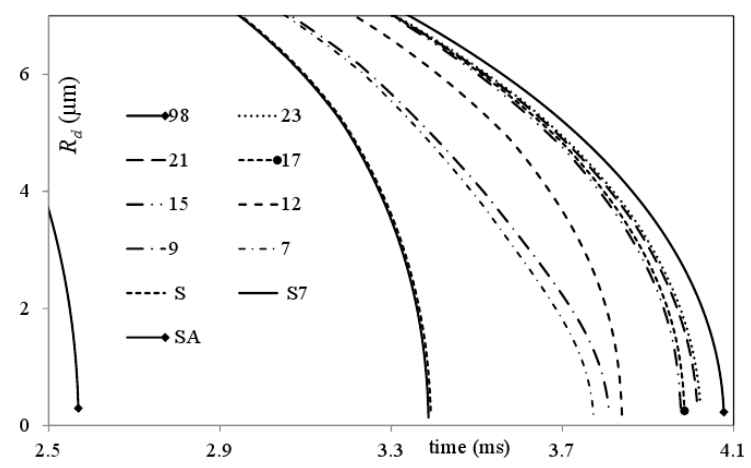

Fig. 3 The same as Fig. 2 but for the droplet radii $R_{d}$. Reprinted from Fuel, Volume 154, Sazhin et al., A multi-dimensional quasi-discrete model for the analysis of Diesel fuel droplet heating and evaporation, Pages 238-266, Copyright Elsevier (2014). 
droplet surface temperature, and an under-estimation of the evaporation time by about $17 \%$. The approximation of Diesel fuel by a single alkane quasi-component $\left(\mathrm{C}_{14.763} \mathrm{H}_{31.526}\right)$ (plots $\mathrm{SA}$ ) leads to under-prediction of the evaporation time by about $37 \%$ which is not acceptable even for qualitative analysis of the process. The plots S and S7 (ignoring the contribution of diaromatic and phenanthrene) for surface temperatures and radii are almost indistinguishable. Also, plots 9 and 7 (ignoring the contribution of diaromatics and phenanthrenes) are rather close. The same applies to plots 23 and 21 (ignoring the contribution of diaromatics and phenanthrenes). This means that the contribution of diaromatics and phenanthrenes can be safely ignored in the approximation of Diesel fuel when modelling the heating and evaporation of fuel droplets in realistic Diesel engine-like conditions. Both for droplet surface temperatures and radii, the accuracy of approximations improves as the number of quasi-components/components (QC/Cs) increases. In the case of 15 QC/Cs the droplet evaporation time can be estimated with an error of about $2.5 \%$. In the case of $21 \mathrm{QC} / \mathrm{Cs}$, this error reduces to about $1.5 \%$. This error is comparable with that for the approximation of Diesel fuel with 40 QC/Cs. Thus when balancing simplicity with accuracy of the model we can recommend the approximation of Diesel fuel with $21 \mathrm{QC} / \mathrm{Cs}$ if errors less than about $2 \%$ can be tolerated. This number of QC/Cs can be reduced to 15 if errors less than about $3 \%$ can be tolerated. The latter model requires about 6 times less CPU time compared with the model taking into account the contributions of all 98 components.

The application of the MDQDM to gasoline fuel droplets and a mixture of biodiesel/Diesel fuel droplets was considered in [79, 80].

The analysis of heating and evaporation of multi-component droplets thus far described has focused primarily on the liquid phase. It has been assumed that all vapour components in the gas phase behave as a single component. This assumption was relaxed in a number of papers, some results of which are summarised below.

In the classical Stefan-Fuchs theory, Equation (15) for evaporation of monocomponent droplets was derived taking into account the conservation of vapour mass flux at any point around a stationary droplet. In the case of multi-component droplets we can impose a similar condition for all individual components in the gas phase. Following [81], this condition can be presented as:

$$
\frac{\mathrm{d}}{\mathrm{d} R}\left(R^{2} \rho_{\text {total }} U Y_{k}-R^{2} D^{(k, m)} \rho_{\text {total }} \frac{\mathrm{d} Y_{k}}{\mathrm{~d} R}\right)=0,
$$

where subscript ${ }_{k}$ refers to ambient gas $(k=0)$ or fuel vapour species $(k=1, \ldots \ldots, n)$, $R \geq R_{d}$ is the distance from the centre of the droplet in the gaseous phase, $D^{(k, m)}$ is the mass diffusion coefficient for the species $k$ in the mixture, $Y_{k}$ are mass fractions of species $k, U$ is the Stefan velocity estimated as

$$
U=\frac{\sum_{k=1}^{n} \dot{m}_{d}^{(k)}}{4 \pi R^{2} \rho_{\mathrm{total}}}
$$


$\dot{m}_{d}^{(k)}$ is the evaporation rate of species $k$ (following [81] and in contrast to Equation (15) we assume that $\dot{m}_{d}^{(k)} \geq 0$ during the evaporation process), $\rho_{\text {total }}$ is the total density of the mixture, including ambient gas.

The analysis of Equation (54) is difficult due to the fact that both $\rho_{\text {total }}$ and $D^{(k, m)}$ are unknown functions of $R$. Our further analysis is based on the assumption that $\rho_{\text {total }}$ and $D^{(k, m)}$ remain constant for all $R$ (the assumption that $\rho_{\text {total }}$ is constant was made when deriving Equation (15)). The values of $D^{(k, m)}$ were estimated in the reference conditions as (Blanc's law):

$$
D^{(k, m)}=\left(\sum_{j=0 ; j \neq k}^{n} \frac{Y_{j(\mathrm{ref})}}{D^{(k, j)}}\right)^{-1},
$$

where

$$
Y_{j(\mathrm{ref})}=\frac{2 Y_{j(\mathrm{~s})}+Y_{j(\infty)}}{3},
$$

$Y_{j(\mathrm{~s})}$ and $Y_{j(\infty)}$ are the mass fractions of species $j$ at the surface of the droplets and in ambient gas, respectively. Expression (57) allows us to consider $\rho_{\text {total }}$ under the reference conditions as well $\left(\rho_{\text {total }}=\rho_{\text {ref }}\right)$.

Having introduced new variable $\zeta=R_{d} / R$, the general analytical solution to Equation (54) was obtained in the form [81]:

$$
Y_{k}=\alpha_{k} \exp \left[-\frac{\dot{m}_{d}^{\text {(total })}}{4 \pi \rho_{\text {total }} R_{d} D^{(k, m)}} \zeta\right]+\varepsilon_{k},
$$

where $\dot{m}_{d}^{(\text {total })}=\sum_{k=1}^{n} \dot{m}_{d}^{(k)}$,

$$
\varepsilon_{k}=\frac{\dot{m}_{d}^{(k)}}{\sum_{k=1}^{n} \dot{m}_{d}^{(k)}}
$$

is the evaporation rate of species $k, \alpha_{k}$ are unknown constants.

Recalling that $Y_{k}(\zeta=0)=Y_{k \infty}$, we find that $\alpha_{k}=Y_{k \infty}-\varepsilon_{k}$. This relation for $\alpha_{k}$ allows us to rewrite Equation (58) for the droplet surface $(\zeta=1)$ as:

$$
Y_{k s}=\left(Y_{k \infty}-\varepsilon_{k}\right) \exp \left[-\frac{\dot{m}_{d}^{(\text {total })}}{4 \pi \rho_{\mathrm{total}} R_{d} D^{(k, m)}}\right]+\varepsilon_{k} .
$$

Equation (58) was rearranged as [81]:

$$
\varepsilon_{k}=\frac{Y_{k s}-Y_{k \infty} \exp \left[-\frac{\dot{m}_{d}^{\text {(total })}}{4 \pi \rho_{\text {total }} R_{d} D^{(k, m)}}\right]}{1-\exp \left[-\frac{\dot{m}_{d}^{\text {total })}}{4 \pi \rho_{\text {total }} R_{d} D^{(k, m)}}\right]},
$$




$$
\sum_{k=1}^{n} \frac{Y_{k s}-Y_{k \infty}}{\left(1-\exp \left[-\frac{\dot{m}_{d}^{\text {(total })}}{4 \pi \rho_{\text {total }} R_{d} D^{(k, m)}}\right]\right)}=1=\sum_{k=1}^{n} Y_{k \infty}
$$

Non-linear Equation (62) was used in [81] to calculate the total evaporation rate $\dot{m}_{d}^{\text {(total) }}$ assuming that the values of all other parameters in this equation are known. Once the value of $\dot{m}_{d}^{\text {(total) }}$ was obtained, the values of $\varepsilon_{k}$ were calculated from Equation (61).

Equation (54) could be formulated in terms of molar rather than mass fluxes [82]. The latter equation could be solved under the assumption that the molar density of the mixture does not depend on the distance from the droplet surface. The solution to this equation would be rather similar to (61) and (62) and its explicit form was given in [82]. These two equations and their solutions predict slightly different evaporation rates since the conditions of constant total mass density and constant molar density of the mixture are not equivalent.

To take into account the effects of multi-component droplet movement on droplet heating and evaporation, in [81] (as well as in a number of other papers, e.g. [83]) it was assumed that there is no interaction between evaporating species. For each of these species the Abramzon and Sirignano model [32] was applied. The validity of this assumption is not at first evident, and this is the reason why, in many papers and books, including [2], the effect of relative motion between species in the gas phase has been ignored altogether.

The authors of $[84,85,86,82]$ drew attention to the fact that more accurate description of multi-component diffusion, compared with Equation (54), should be based on the Maxwell-Stefan equations. Ignoring the Soret effects, diffusion due to pressure gradients and external forces, these equations can be presented as [87, 82]:

$$
\nabla X^{(p)}=\sum_{k=0}^{n} \frac{1}{C_{m} D_{p k}}\left(X^{(p)} \mathbf{N}^{(k)}-X^{(k)} \mathbf{N}^{(p)}\right),
$$

where $X^{(k)}$ is the molar fraction of the $k$ th component, $C_{m}$ is the molar density of the mixture, $D_{p k}=D_{k p}$ is the binary diffusion coefficient of the $p$ th component into the $k$ th component, $\mathbf{N}^{(p)}$ is the molar flux of the $p$ th component, $k=0$ refers to ambient gas.

For a multi-component spherical droplet only the radial components of the species molar fluxes can be retained. In this case, Eq. (63) was presented in a similar format to that inferred from Eq. (54). This allowed the authors of [82] to present the solution to (63) in a similar format to (61) and (62), but for molar fractions, assuming that the total molar density does not depend on the distance from the droplet surface. It was shown that the predictions based on Eq. (54) (Stefan-Fuchs equation) underestimate the total evaporation rate, especially at high ambient gas temperatures, for various droplet compositions. The largest deviation of the absolute values of the evaporation rate, predicted by the Stefan-Fuchs and Maxwell-Stefan equations, was found when none of the species mass fractions was dominant. 
A new quasi-dimensional multi-component heating and evaporation model for multi-component fuel droplets was suggested in [88]. In contrast to the Discrete Component Model, this model is based not on the rigorous solution to heat transfer and species diffusion equations inside droplets, but on the polynomial (quadratic) approximations of the temperature and mass fractions of species distributions inside droplets.

\section{Kinetic and molecular dynamics models}

So far the modelling of droplet heating and evaporation processes has been based on the hydrodynamic approximation. In this approximation, vapour at the droplet surface is assumed to be saturated and the evaporation is modelled as the diffusion of vapour from the droplet surface to the ambient gas [2]. The limitations of this approximation have been well-known since the pioneering papers published more than 100 years ago (see [89] and the references therein). In a number of studies, summarised in [2], the heating and evaporation of n-dodecane $\left(\mathrm{C}_{12} \mathrm{H}_{26}\right)$ (a crude approximation for Diesel fuel) and a mixture of n-dodecane (approximating alkanes in Diesel fuel) and p-dipropylbenzene (approximating aromatics in Diesel fuel) droplets was studied and a new model combining the kinetic and hydrodynamic approaches was developed. ${ }^{3}$ In the immediate vicinity of droplet surfaces (up to about one hundred molecular mean free paths), the vapour and ambient gas dynamics were studied based on the solution to the Boltzmann equation (kinetic region), while at larger distances the analysis was based on the hydrodynamic equations (hydrodynamic region). Mass, momentum and energy fluxes were conserved at the interface between these regions and between the kinetic region and liquid. The modelling took into account the contributions of up to three components in the kinetic region (up to two components approximating Diesel fuel, and air approximated by nitrogen). The above-mentioned three regions in the vicinity of the droplet surface are schematically shown in Fig. 4. In kinetic modelling inelastic collisions between molecules were taken into account using a simplified model (see [2] for details).

The boundary conditions at the interfaces between the kinetic and hydrodynamic regions were inferred based on the requirement of the conservation of heat and mass fluxes at this interface. The hydrodynamic heat and mass fluxes were calculated based on the simplifying assumptions that the temperature at the outer boundary of the kinetic region is equal to the droplet surface temperature and vapour pressure at this boundary is equal to the saturated vapour pressure at a temperature equal to the droplet surface temperature. The requirement of the conservation of heat and mass fluxes at this interface allowed us to find the corrected values of temperature and vapour density. The main problem with this approach is that the heat and mass fluxes in the hydrodynamic region, calculated based on these corrected values of temperature and vapour density, are not equal to the heat and mass fluxes in

3 An approximation of Diesel fuel by a mixture of $n$-dodecane and $m$-xylene was considered in [90], but the implications of this approximation for kinetic modelling have not been investigated. 


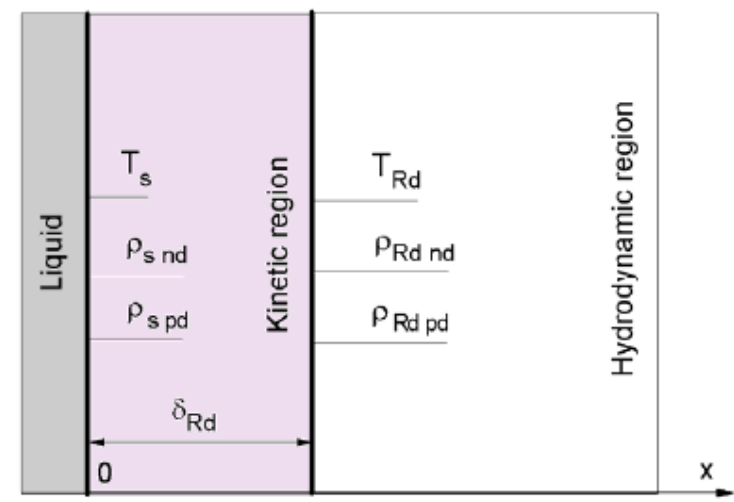

Fig. 4 Liquid, kinetic and hydrodynamic regions near the surface of the droplet. $T_{s}$ is the droplet surface temperature, $\rho_{s(n d, p d)}$ are n-dodecane $(n d)$ and p-dipropylbenzene $(p d)$ vapour densities in the immediate vicinity of the droplet surface, $T_{R d}$ and $\rho_{R d(n d, p d)}$ are the temperature and ndodecane $\left.{ }_{n d}\right)$ and p-dipropylbenzene $\left(_{p d}\right)$ vapour densities at the outer boundary of the kinetic region. Reprinted from International Journal of Heat and Mass Transfer, Volume 93, Sazhin et al., A self-consistent kinetic model for droplet heating and evaporation, Pages 1206-1217, Copyright Elsevier (2016).

the hydrodynamic region used to find these corrected values, in the general case. This problem was resolved in [91], in which a new self-consistent kinetic model for droplet heating and evaporation is described.

As mentioned earlier, the solution to the Boltzmann equation in the kinetic region requires formulation of the boundary condition at the liquid/gas interface. This boundary condition is essentially controlled by the evaporation coefficient. An approximation of the results of molecular dynamics calculations of this coefficient, using the United Atom Model (UAM), is given by the following equation:

$$
\beta_{e}\left(T_{s}\right)=7 \times 10^{-6} T_{s}^{2}-9.8 \times 10^{-3} T_{s}+3.7215 .
$$

where $T_{S}$ is the droplet surface temperature.

One of the main limitations of the UAM, used for approximation (64), is that in this model the interaction between individual molecules was described using the force field (FF) methods, which simplify both inter- and inner-molecular interactions by ignoring electrons per se. The applicability of this approach is far from obvious, as the dynamics of individual molecules in the vicinity of droplet surfaces are essentially quantum mechanical processes. The quantum mechanical (quantumchemical (QC)) models describing the processes at and in the vicinity of Diesel fuel droplet surfaces are described in $[92,93,94,95,96]$.

It was shown that the most efficient approach to taking into account quantum chemical effects on the value of the evaporation coefficients could be based on the transition state theory (TST) and quantum chemical DFT methods [94]. These were 
applied to several ensembles of n-dodecane conformers. There was similarity between the approach used in [94] and the one used previously (see [2]). In contrast to the previous studies, however, in the analysis of [94] the TST was based on a QC DFT approach taking into account the conformerisation of n-dodecane molecules (considered to be representative of Diesel fuel). It was shown that the most accurate expression for the condensation coefficient is the one averaged over the states of various conformers transferred between two phases [94]:

$\beta_{e}=\left\{1-\left[\frac{\rho_{g}}{\rho_{l}} \exp \frac{\left\langle\Delta G_{g \rightarrow l}\right\rangle}{R_{u} T}\right]^{1 / 3}\right\} \exp \left\{-0.5\left[\left[\frac{\rho_{g}}{\rho_{l}} \exp \frac{\left\langle\Delta G_{g \rightarrow l}\right\rangle}{R_{u} T}\right]^{1 / 3}-1\right]^{-1}\right\}$,

where $R_{u}$ is the universal gas constant, $\rho_{g(l)}$ is the gas (liquid) density, $\Delta G_{g \rightarrow l}$ is the change in the Gibbs free energy during the condensation process. It was assumed that the process under consideration is quasi-steady-state and the condensation coefficient is equal to the evaporation coefficient.

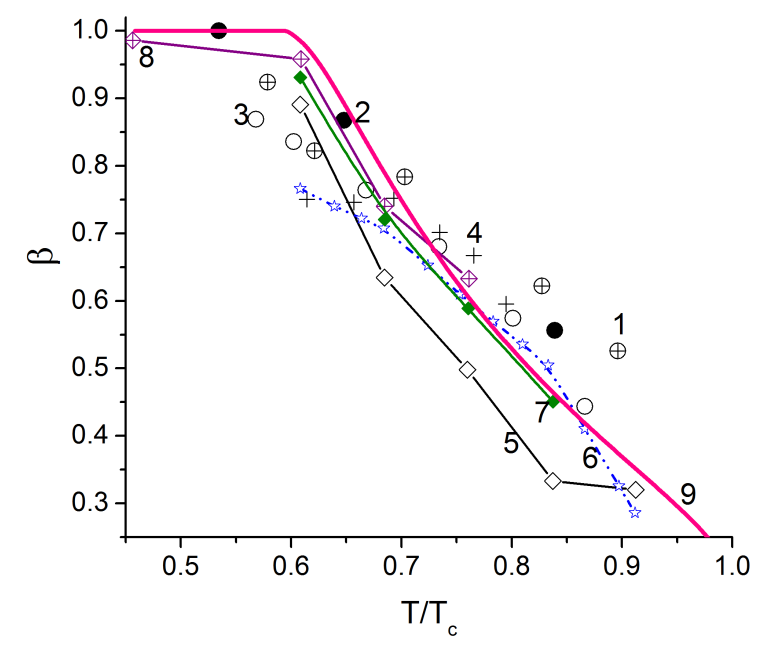

Fig. 5 The values of the evaporation coefficient $\beta=\beta_{e}$, predicted by MD, FF (symbols 1-4, curves 5-8) and Formula (65) (curve 9), versus normalised temperature $\left(T / T_{c}\right.$, where $T_{c}$ is the critical temperature). Symbols (1-4) refer to the models for structureless LJ fluids with various input parameters [97, 98], curves 5 and 7 refer to the results obtained based on the UAM reported in $[99,100]$, respectively, curve 6 refers to the results of calculations based on the TST model reproduced from [99], curve 8 is based on the results of calculations using the model described by Mizuguchi et al. [98]. QC calculations were performed using DFT, $\omega$ B97X-D/cc-pVTZ and SMD/ $\omega$ B97X-D/cc-pVTZ. Reprinted from Fuel, Volume 165, Sazhin et al., Quantum-chemical analysis of the processes at the surfaces of Diesel fuel droplets, Pages 405-412, Copyright Elsevier (2016). 
The effects of both the conformerisation and cross-conformerisation (changes in conformer state during transfer into another phase) of n-dodecane molecules (CDM effects), which can contribute to the Gibbs free energies of evaporation and solvation, were taken into account. Ninety-five stable conformers were selected based on the changes in the Gibbs free energy.

A comparison between the results of calculations of $\beta_{e}$ based on Expression (65) and those obtained previously is shown in Fig. 5. As can be seen from this figure, taking into account the QC effects leads to marginal modification of the predicted evaporation/condensation coefficients, except at temperatures close to the critical temperature (where this modification turned out to be significant). Thus, although the analysis of the QC effects takes into account many new effects ignored in the conventional FF approach, the contribution of these effects to the values of the evaporation/condensation coefficient turned out to be marginal, unless temperatures close to the critical temperature were considered.

\section{Acknowledgements}

The author is grateful to EPSRC (grants EP/K005758/1, EP/K020528/1 and EP/M002608/1) for their financial support.

\section{References}

1. S. S. Sazhin, Advanced models of fuel droplet heating and evaporation, Progress in Energy and Combustion Science 32 (2006) 162-214.

2. S. S. Sazhin, Droplets and Sprays, Springer, Heidelberg, 2014.

3. S. S. Sazhin, Modelling of fuel droplet heating and evaporation: Recent results and unsolved problems, Fuel 196 (2017) 69 - 101. doi:https://doi.org/10.1016/j.fuel.2017.01.048.

URL http://www.sciencedirect.com/science/article/pii/S0016236117300583

4. J. B. Heywood, Internal Combustion Engines Fundamentals, McGraw-Hill, New York, 1988.

5. M. Rahman, M. Saghir, Thermodiffusion or Soret effect: Historical review, International Journal of Heat and Mass Transfer 73 (2014) 693 - 705. doi:http://dx.doi.org/10.1016/j.ijheatmasstransfer.2014.02.057.

URL http://www.sciencedirect.com/science/article/pii/S0017931014001859

6. W. A. Sirignano, Advances in droplet array combustion theory and modeling, Progress in Energy and Combustion Science 42 (2014) 54 - 86. doi:http://dx.doi.org/10.1016/j.pecs.2014.01.002.

URL http://www.sciencedirect.com/science/article/pii/S0360128514000033

7. S. K. Aggarwal, Single droplet ignition: Theoretical analyses and experimental findings, Progress in Energy and Combustion Science 45 (2014) 79 - 107. doi:http://dx.doi.org/10.1016/j.pecs.2014.05.002.

URL http://www.sciencedirect.com/science/article/pii/S0360128514000276

8. A. K. Yadav, A. Chowdhury, A. Srivastava, Interferometric investigation of methanol droplet combustion in varying oxygen environments under normal gravity, International Journal of Heat and Mass Transfer 111 (2017) 871 - 883. doi:https://doi.org/10.1016/j.ijheatmasstransfer.2017.03.125.

URL http://www.sciencedirect.com/science/article/pii/S0017931016341424 
9. C. Wang, R. Xu, Y. Song, P. Jiang, Study on water droplet flash evaporation in vacuum spray cooling, International Journal of Heat and Mass Transfer 112 (2017) $279-288$. doi:https://doi.org/10.1016/j.ijheatmasstransfer.2017.04.111.

URL http://www.sciencedirect.com/science/article/pii/S0017931017303319

10. K. Wittig, P. Nikrityuk, A. Richter, Drag coefficient and Nusselt number for porous particles under laminar flow conditions, International Journal of Heat and Mass Transfer 112 (2017) 1005 - 1016. doi:https://doi.org/10.1016/j.ijheatmasstransfer.2017.05.035. URL http://www.sciencedirect.com/science/article/pii/S0017931017305604

11. B. T. Bojko, P. E. DesJardin, On the development and application of a droplet flameletgenerated manifold for use in two-phase turbulent combustion simulations, Combustion and Flame 183 (2017) 50 - 65. doi:https://doi.org/10.1016/j.combustflame.2017.04.029. URL http://www.sciencedirect.com/science/article/pii/S0010218017301645

12. H. Olguin, E. Gutheil, Influence of evaporation on spray flamelet structures, Combustion and Flame 161 (4) (2014) 987 - 996. doi:http://dx.doi.org/10.1016/j.combustflame.2013.10.010. URL http://www.sciencedirect.com/science/article/pii/S0010218013003842

13. O. Rybdylova, M. Al Qubeissi, M. Braun, C. Crua, J. Manin, L. M. Pickett, G. de Sercey, E. M. Sazhina, S. S. Sazhin, M. Heikal, A model for droplet heating and its implementation into ANSYS Fluent, International Communications in Heat and Mass Transfer 76 (2016) 265-270.

14. G. J. Brereton, A discrete multicomponent temperature-dependent model for the evaporation of spherical droplets, International Journal of Heat and Mass Transfer 60 (2013) 512-522.

15. A. Y. Snegirev, Transient temperature gradient in a single-component vaporizing droplet, International Journal of Heat and Mass Transfer 65 (2013) 80-94.

16. H. Xiao, L. Zhao, Z. Li, M. Wei, G. Guo, Development of a simplified model for droplet vaporization, Thermal Science 20 (1) (2016) 337-345.

17. V. R. Subramanian, V. D. Diwakar, D. Tapriyal, Efficient macro-micro scale coupled modeling of batteries, Journal of the Elecrochemical Society 152 (10) (2005) A2002-A2008.

18. A. Snegirev, V. A. Talalov, A. S. Tsoi, S. S. Sazhin, C. Crua, Advancement in turbulent spray modeling: the effect of internal temperature gradient in droplets, Proceedings of International Symposium on Advances in Computational Heat Transfer (16 July Bath, 2012, UK) CHT12MP09.

19. T. J. Moore, M. R. Jones, Solving nonlinear heat transfer problems using variation of parameters, International Journal of Thermal Sciences 93 (2015) 29 - 35. doi:http://dx.doi.org/10.1016/j.ijthermalsci.2015.02.002. URL http://www.sciencedirect.com/science/article/pii/S1290072915000538

20. M. Mierzwiczak, W. Chen, Z.-J. Fu, The singular boundary method for steadystate nonlinear heat conduction problem with temperature-dependent thermal conductivity, International Journal of Heat and Mass Transfer 91 (2015) 205 - 217. doi:http://dx.doi.org/10.1016/j.ijheatmasstransfer.2015.07.051.

URL http://www.sciencedirect.com/science/article/pii/S0017931015007711

21. J. Hristov, An approximate analytical (integral-balance) solution to a non-linear heat diffusion equation, Thermal Science 19 (2) (2015) 723-733.

22. S. Feng, X. Cui, A. Li, Fast and efficient analysis of transient nonlinear heat conduction problems using combined approximations (CA) method, International Journal of Heat and Mass Transfer 97 (2016) 638 - 644. doi:http://dx.doi.org/10.1016/j.ijheatmasstransfer.2016.02.061. URL http://www.sciencedirect.com/science/article/pii/S0017931015316550

23. H. Qi, X. Guo, Transient fractional heat conduction with generalized Cattaneo model, International Journal of Heat and Mass Transfer 76 (2014) 535 - 539. doi:http://dx.doi.org/10.1016/j.ijheatmasstransfer.2013.12.086. URL http://www.sciencedirect.com/science/article/pii/S0017931014003858

24. R. Khayat, J. deBruyn, M. Niknami, D. Stranges, R. Khorasany, Non-Fourier effects in macro- and micro-scale non-isothermal flow of liquids and gases. review, International Journal of Thermal Sciences 97 (2015) 163 - 177. 
doi:http://dx.doi.org/10.1016/j.ijthermalsci.2015.06.007.

URL http://www.sciencedirect.com/science/article/pii/S1290072915001726

25. L. Zhang, X. Shang, Analytical solution to non-Fourier heat conduction as a laser beam irradiating on local surface of a semi-infinite medium, International Journal of Heat and Mass Transfer 85 (2015) 772 - 780. doi:http://dx.doi.org/10.1016/j.ijheatmasstransfer.2015.02.024.

URL http://www.sciencedirect.com/science/article/pii/S0017931015001878

26. V. Borukhov, G. Zayats, Identification of a time-dependent source term in nonlinear hyperbolic or parabolic heat equation, International Journal of Heat and Mass Transfer 91 (2015) 1106 - 1113. doi:http://dx.doi.org/10.1016/j.ijheatmasstransfer.2015.07.066.

URL http://www.sciencedirect.com/science/article/pii/S0017931015007863

27. K. Zhukovsky, Exact solution of Guyer-Krumhansl type heat equation by operational method, International Journal of Heat and Mass Transfer 96 (2016) 132 - 144. doi:http://dx.doi.org/10.1016/j.ijheatmasstransfer.2016.01.005.

URL http://www.sciencedirect.com/science/article/pii/S0017931015315611

28. K. Zhukovsky, Violation of the maximum principle and negative solutions for pulse propagation in Guyer-Krumhansl model, International Journal of Heat and Mass Transfer 98 (2016) 523 - 529. doi:http://dx.doi.org/10.1016/j.ijheatmasstransfer.2016.03.021.

URL http://www.sciencedirect.com/science/article/pii/S0017931016301971

29. S.-N. Li, B.-Y. Cao, On defects of Taylor series approximation in heat conduction models, International Journal of Heat and Mass Transfer 98 (2016) 824 - 832. doi:http://dx.doi.org/10.1016/j.ijheatmasstransfer.2016.03.067.

URL http://www.sciencedirect.com/science/article/pii/S001793101531749X

30. A. Aissa, M. Abdelouahab, A. Noureddine, M. El Ganaoui, B. Pateyron, Ranz and Marcshall correlations limits on heat flow between a sphere and its surrounding gas at high temperature, Thermal Science 19 (5) (2015) 1521-1528.

31. R. Clift, J. R. Grace, M. E. Weber, Bubbles, Drops and Particles, Academic Press, New York, 1978.

32. B. Abramzon, W. A. Sirignano, Droplet vaporization model for spray combustion calculations, International Journal of Heat and Mass Transfer 32 (1989) 1605-1618.

33. J. Will, N. Kruyt, C. Venner, An experimental study of forced convective heat transfer from smooth, solid spheres, International Journal of Heat and Mass Transfer 109 (2017) 1059 1067. doi:https://doi.org/10.1016/j.ijheatmasstransfer.2017.02.018.

URL http://www.sciencedirect.com/science/article/pii/S0017931016331763

34. V. Borodulin, V. Letushko, M. Nizovtsev, A. Sterlyagov, Determination of parameters of heat and mass transfer in evaporating drops, International Journal of Heat and Mass Transfer 109 (2017) 609-618. doi:https://doi.org/10.1016/j.ijheatmasstransfer.2017.02.042.

URL http://www.sciencedirect.com/science/article/pii/S001793101633304X

35. C. Yin, Modelling of heating and evaporation of n-heptane droplets: Towards a generic model for fuel droplet/particle conversion, Fuel 141 (2015) 64 - 73. doi:http://dx.doi.org/10.1016/j.fuel.2014.10.031.

URL http://www.sciencedirect.com/science/article/pii/S0016236114010266

36. B. Abramzon, S. S. Sazhin, Convective vaporization of fuel droplets with thermal radiation absorption, Fuel 85 (2006) 32-46.

37. G. Gouesbet, G. Grehan, Generalized Lorenz-Mie Theories, Springer, Berlin, 2011.

38. C. Bouchenna, M. A. Saada, S. Chikh, L. Tadrist, Generalized formulation for evaporation rate and flow pattern prediction inside an evaporating pinned sessile drop, International Journal of Heat and Mass Transfer 109 (2017) 482 - 500. doi:https://doi.org/10.1016/j.ijheatmasstransfer.2017.01.114.

URL http://www.sciencedirect.com/science/article/pii/S0017931016317719

39. M. Ashna, M. H. Rahimian, LMB simulation of head-on collision of evaporating and burning droplets in coalescence regime, International Journal of Heat and Mass Transfer 109 (2017) 520 - 536. doi:https://doi.org/10.1016/j.ijheatmasstransfer.2017.01.108.

URL http://www.sciencedirect.com/science/article/pii/S0017931016324309 
40. X. Chen, X. Wang, P. G. Chen, Q. Liu, Thermal effects of substrate on Marangoni flow in droplet evaporation: Response surface and sensitivity analysis, International Journal of Heat and Mass Transfer 113 (2017) 354 - 365. doi:https://doi.org/10.1016/j.ijheatmasstransfer.2017.05.076.

URL http://www.sciencedirect.com/science/article/pii/S0017931016341448

41. S. Tonini, G. E. Cossali, An analytical model of liquid drop evaporation in gaseous environment, International Journal of Thermal Sciences 57 (2012) 45-53.

42. F. Marti, O. Martinez, D. Mazo, J. Garman, D. Dunn-Rankin, Evaporation of a droplet larger than the Kolmogorov length scale immersed in a relative mean flow, International Journal of Multiphase Flow 88 (2017) 63 - 68. doi:http://dx.doi.org/10.1016/j.ijmultiphaseflow.2016.09.019. URL http://www.sciencedirect.com/science/article/pii/S0301932215300604

43. R. N. Dahms, J. C. Oefelein, The significance of drop non-sphericity in sprays, International Journal of Multiphase Flow 86 (2016) $67-85$. doi:http://dx.doi.org/10.1016/j.ijmultiphaseflow.2016.07.010.

URL http://www.sciencedirect.com/science/article/pii/S030193221530104X

44. A. E. Elwardany, I. G. Gusev, G. Castanet, F. Lemoine, S. S. Sazhin, Mono- and multicomponent droplet cooling/heating and evaporation: comparative analysis of numerical models, Atomization and Sprays 21 (2011) 907-931.

45. S. S. Sazhin, M. Al Qubeissi, J.-F. Xie, Two approaches to modelling the heating of evaporated droplets, International Communications in Heat and Mass Transfer 57 (2014) 353-356.

46. E. Michaelides, Particles, Bubbles and Drops, World Scietific, New Jersey, 2006.

47. C. Crua, T. Shoba, M. Heikal, M. Gold, C. Higham, High-speed microscopic imaging of the initial stage of diesel spray formation and primary breakup, SAE International 2010-01-2247 (2010) $1-10$.

48. C. Niven, On the conduction of heat in ellipsoids of revolution, Philosophical Transactions of the Royal Society London 171 (1880) 117-151.

49. M. A. Jog, H. M. A., Transient heat transfer to a spheroidal liquid drop suspended in an electric field, International Journal of Heat and Fluid Flow 18 (1997) 411-418.

50. D. R. Lima, S. N. Farias, G. B. Lima, Mass transport in spheroids using the Galerkin method, Brazilian Journal of Chemical Engineering 21 (2004) 667-680.

51. L. He, D. K. Tafti, Heat transfer in an assembly of ellipsoidal particles at low to moderate Reynolds numbers, International Journal of Heat and Mass Transfer 114 (2017) 324 - 336. doi:https://doi.org/10.1016/j.ijheatmasstransfer.2017.06.068.

URL http://www.sciencedirect.com/science/article/pii/S0017931017312942

52. R. S. Alassar, Forced convection past an oblate spheroid at low to moderate Reynolds numbers, ASME Journal of Heat Transfer 127 (2005) 1062-1070.

53. A. Richter, P. A. Nikrityuk, Drag forces and heat transfer coefficients for spherical, cuboidal and ellipsoidal particles in cross flow at sub-critical Reynolds numbers, International Journal or Heat and Mass Transfer 55 (2012) 1343-1354.

54. N. Kishore, S. Gu, Momentum and heat transfer phenomena of spheroid particles at moderate Reynolds and Prandtl numbers, International Journal of Heat Mass Transfer 55 (2011) 25952601.

55. B. Sreenivasulu, B. Srinivas, K. Ramesh, Forced convection heat transfer from a spheroid to a power law fluid, International Journal of Heat and Mass Transfer 70 (2014) $71-80$. doi:http://dx.doi.org/10.1016/j.ijheatmasstransfer.2013.10.065.

URL http://www.sciencedirect.com/science/article/pii/S0017931013009253

56. B. Sreenivasulu, B. Srinivas, Mixed convection heat transfer from a spheroid to a Newtonian fluid, International Journal of Thermal Sciences 87 (2015) 1 - 18. doi:http://dx.doi.org/10.1016/j.ijthermalsci.2014.08.002.

URL http://www.sciencedirect.com/science/article/pii/S1290072914002270

57. G. Juncu, Unsteady heat transfer from an oblate/prolate spheroid, International Journal of Heat Mass Transfer 53 (2010) 3483-3494.

58. D. A. Grow, Heat and mass transfer to an elliptical particle, Combustion and Flame 80 (1990) 209-213. 
59. S. Tonini, G. E. Cossali, An exact solution of the mass transport equations for spheroidal evaporating drops, International Journal of Heat and Mass Transfer 60 (2013) 236-240.

60. J. Li, J. Zhang, A theoretical study of the spheroidal droplet evaporation in forced convection, Physics Letters A 378 (47) (2014) 3537 - 3543. doi:http://dx.doi.org/10.1016/j.physleta.2014.10.020.

URL http://www.sciencedirect.com/science/article/pii/S037596011401038X

61. G. Strotos, I. Malgarinos, N. Nikolopoulos, M. Gavaises, Predicting the evaporation rate of stationary droplets with the VOF methodology for a wide range of ambient temperature conditions, International Journal of Thermal Sciences 109 (2016) 253 - 262. doi:http://dx.doi.org/10.1016/j.ijthermalsci.2016.06.022.

URL http://www.sciencedirect.com/science/article/pii/S1290072915302738

62. S. Tonini, G. Cossali, An evaporation model for oscillating spheroidal drops, International Communications in Heat and Mass Transfer 51 (2014) 18 - 24. doi:http://dx.doi.org/10.1016/j.icheatmasstransfer.2013.12.001.

URL http://www.sciencedirect.com/science/article/pii/S0735193313002388

63. F. Mashayek, Dynamics of evaporating drops. part ii: free oscillations, International Journal of Heat and Mass Transfer 44 (8) (2001) 1527-1541.

64. S. Tonini, G. Cossali, One-dimensional analytical approach to modelling evaporation and heating of deformed drops, International Journal of Heat and Mass Transfer 97 (2016) 301 307. doi:http://dx.doi.org/10.1016/j.ijheatmasstransfer.2016.02.004.

URL http://www.sciencedirect.com/science/article/pii/S0017931015317415

65. C. Sasmal, N. Nirmalkar, Momentum and heat transfer characteristics from heated spheroids in water based nanofluids, International Journal of Heat and Mass Transfer 96 (2016) 582 601. doi:http://dx.doi.org/10.1016/j.ijheatmasstransfer.2016.01.054.

URL http://www.sciencedirect.com/science/article/pii/S0017931015307225

66. V. S. Zubkov, G. E. Cossali, S. Tonini, O. Rybdylova, C. Crua, M. Heikal, S. S. Sazhin, Mathematical modelling of heating and evaporation of a spheroidal droplet, International Journal of Heat and Mass Transfer 108, Part B (2017) 2181 - 2190. doi:https://doi.org/10.1016/j.ijheatmasstransfer.2016.12.074.

URL http://www.sciencedirect.com/science/article/pii/S001793101633438X

67. L. Liu, Y. Liu, M. Mi, Z. Wang, L. Jiang, Evaporation of a bicomponent droplet during depressurization, International Journal of Heat and Mass Transfer 100 (2016) 615 - 626. doi:http://dx.doi.org/10.1016/j.ijheatmasstransfer.2016.05.007. URL http://www.sciencedirect.com/science/article/pii/S0017931015314897

68. P. Yi, W. Long, L. Feng, W. Wang, C. Liu, An experimental and numerical study of the evaporation and pyrolysis characteristics of lubricating oil droplets in the natural gas engine conditions, International Journal of Heat and Mass Transfer 103 (2016) 646 - 660. doi:http://dx.doi.org/10.1016/j.ijheatmasstransfer.2016.07.084.

URL http://www.sciencedirect.com/science/article/pii/S0017931016309991

69. S. Srivastava, F. Jaberi, Large eddy simulations of complex multicomponent diesel fuels in high temperature and pressure turbulent flows, International Journal of Heat and Mass Transfer 104 (2017) 819 - 834. doi:http://dx.doi.org/10.1016/j.ijheatmasstransfer.2016.07.011. URL http://www.sciencedirect.com/science/article/pii/S0017931016304392

70. S. S. Sazhin, M. Al Qubeissi, R. Kolodnytska, A. Elwardany, R. Nasiri, M. Heikal, Modelling of biodiesel fuel droplet heating and evaporation, Fuel 115 (2014) 559-572.

71. M. A. Qubeissi, S. S. Sazhin, C. Crua, J. Turner, M. R. Heikal, Modelling of biodiesel fuel droplet heating and evaporation: Effects of fuel composition, Fuel 154 (2015) 308 - 318. doi:http://dx.doi.org/10.1016/j.fuel.2015.03.051.

URL http://www.sciencedirect.com/science/article/pii/S0016236115003476

72. C. Laurent, G. Lavergne, P. Villedieu, Continuous thermodynamics for droplet vaporization: Comparison between Gamma-PDF model and QMoM, Comptes Rendus Mecanique 337 (6) (2009) 449 - 457. doi:http://dx.doi.org/10.1016/j.crme.2009.06.004.

URL http://www.sciencedirect.com/science/article/pii/S1631072109000680

73. S. L. Singer, Direct quadrature method of moments with delumping for modeling multicomponent droplet vaporization, International Journal of Heat and Mass Transfer 103 (2016) 940 
- 954. doi:http://dx.doi.org/10.1016/j.ijheatmasstransfer.2016.07.067.

URL http://www.sciencedirect.com/science/article/pii/S0017931016309450

74. S. S. Sazhin, A. Elwardany, E. M. Sazhina, M. R. Heikal, A quasi-discrete model for heating and evaporation of complex multicomponent hydrocarbon fuel droplets, International Journal of Heat and Mass Transfer 54 (2011) 4325-4332.

75. A. E. Elwardany, S. S. Sazhin, A quasi-discrete model for droplet heating and evaporation: Application to Diesel and gasoline fuels, Fuel 97 (2012) 685-694.

76. S. S. Sazhin, M. Al Qubeissi, R. Nasiri, V. M. Gunko, A. E. Elwardany, F. Lemoine, F. Grisch, M. R. Heikal, A multi-dimensional quasi-discrete model for the analysis of Diesel fuel droplet heating and evaporation, Fuel 129 (2014) 238-266.

77. I.-P. Kanaveli, M. Atzemi, E. Lois, Predicting the viscosity of diesel/biodiesel blends, Fuel 199 (2017) 248 - 263. doi:https://doi.org/10.1016/j.fuel.2017.02.077.

URL http://www.sciencedirect.com/science/article/pii/S0016236117302272

78. M. Lapuerta, J. Rodrguez-Fernndez, D. Fernndez-Rodrguez, R. Patio-Camino, Modeling viscosity of butanol and ethanol blends with diesel and biodiesel fuels, Fuel 199 (2017) 332 338. doi:https://doi.org/10.1016/j.fuel.2017.02.101.

URL http://www.sciencedirect.com/science/article/pii/S0016236117302521

79. M. A. Qubeissi, S. S. Sazhin, J. Turner, S. Begg, C. Crua, M. Heikal, Modelling of gasoline fuel droplets heating and evaporation, Fuel 159 (2015) 373-384.

80. M. A. Qubeissi, S. S. Sazhin, A. E. Elwardany, Modelling of blended Diesel and biodiesel fuel droplet heating and evaporation, Fuel 187 (2017) 349 - 355 . doi:https://doi.org/10.1016/j.fuel.2016.09.060.

URL http://www.sciencedirect.com/science/article/pii/S0016236116309176

81. S. Tonini, G. E. Cossali, A novel formulation of multi-component drop evaporation models for spray applications, International Journal of Thermal Sciences 89 (2015) 245 - 253. doi:http://dx.doi.org/10.1016/j.ijthermalsci.2014.10.016.

URL http://www.sciencedirect.com/science/article/pii/S1290072914003007

82. S. Tonini, G. Cossali, A multi-component drop evaporation model based on analytical solution of Stefan-Maxwell equations, International Journal of Heat and Mass Transfer 92 (2016) 184 - 189. doi:http://dx.doi.org/10.1016/j.ijheatmasstransfer.2015.08.014. URL http://www.sciencedirect.com/science/article/pii/S0017931015301551

83. X. Ma, F. Zhang, K. Han, G. Song, Numerical modeling of acetone-butanolethanol and diesel blends droplet evaporation process, Fuel 174 (2016) 206 - 215. doi:http://dx.doi.org/10.1016/j.fuel.2016.01.091.

URL http://www.sciencedirect.com/science/article/pii/S0016236116001125

84. N. Padoin, A. T. D. Toe, L. P. Rangel, K. Ropelato, C. Soares, Heat and mass transfer modeling for multicomponent multiphase flow with CFD, International Journal of Heat and Mass Transfer 73 (2014) 239 - 249. doi:http://dx.doi.org/10.1016/j.ijheatmasstransfer.2014.01.075.

URL http://www.sciencedirect.com/science/article/pii/S0017931014001161

85. G. Jarvas, J. Kontos, J. Hancsok, A. Dallos, Modeling ethanol-blended gasoline droplet evaporation using COSMO-RS theory and computation fluid dynamics, International Journal of Heat and Mass Transfer 84 (2015) 1019 - 1029. doi:http://dx.doi.org/10.1016/j.ijheatmasstransfer.2014.12.046.

URL http://www.sciencedirect.com/science/article/pii/S0017931014011545

86. A. T. O. D. Toe, N. Padoin, K. Ropelato, C. Soares, Cross diffusion effects in the interfacial mass and heat transfer of multicomponent droplets, International Journal of Heat and Mass Transfer 85 (2015) 830 - 840. doi:http://dx.doi.org/10.1016/j.ijheatmasstransfer.2015.01.131.

URL http://www.sciencedirect.com/science/article/pii/S0017931015001490

87. R. B. Bird, W. E. Stewart, E. N. Lightfoot, Transport Phenomena, John Wiley \& Sons, Inc., New York, 2002.

88. P. Yi, W. Long, M. Jia, J. Tian, B. Li, Development of a quasi-dimensional vaporization model for multi-component fuels focusing on forced convection and high temperature conditions, International Journal of Heat and Mass Transfer 97 (2016) 130 - 145. 
doi:http://dx.doi.org/10.1016/j.ijheatmasstransfer.2016.01.075.

URL http://www.sciencedirect.com/science/article/pii/S0017931015308760

89. N. A. Fuchs, Evaporation and Droplet Growth in Gaseous Media, Pergamon Press, London, 1959.

90. R. Payri, J. P. Viera, Y. Pei, S. Som, Experimental and numerical study of lift-off length and ignition delay of a two-component diesel surrogate, Fuel 158 (2015) 957 - 967. doi:http://dx.doi.org/10.1016/j.fuel.2014.11.072.

URL http://www.sciencedirect.com/science/article/pii/S0016236114011764

91. S. S. Sazhin, I. N. Shishkova, M. Al Qubeissi, A self-consistent kinetic model for droplet heating and evaporation, International Journal of Heat and Mass Transfer 93 (2016) 12061217.

92. V. M. Gunko, R. Nasiri, S. S. Sazhin, F. Lemoine, F. Grisch, A quantum chemical study of the processes during the evaporation of real-life diesel fuel droplets, Fluid Phase Equilibria 356 (2013) 146-156.

93. V. M. Gunko, R. Nasiri, S. S. Sazhin, A study of the evaporation and condensation of nalkane clusters and nanodroplets using quantum chemical methods, Fluid Phase Equilibria 366 (2014) 99-107.

94. V. M. Gunko, R. Nasiri, S. S. Sazhin, Effects of the surroundings and conformerisation of n-dodecane molecules on evaporation/condensation processes, Journal of Chemical Physics 142 (3) (2015) 034502.

95. R. Nasiri, V. M. Gunko, S. S. Sazhin, The effects of internal molecular dynamics on the evaporation/condensation of n-dodecane, Theoretical Chemistry Accounts 134 (2015) N83.

96. S. S. Sazhin, V. M. Gunko, R. Nasiri, Quantum-chemical analysis of the processes at the surfaces of diesel fuel droplets, Fuel 165 (2016) 405-412.

97. A. Lotfi, J. Vrabec, J. Fischer, Evaporation from a free liquid surface, International Journal of Heat and Mass Transfer 73 (2014) 303-317.

98. H. Mizuguchi, G. Nagayama, T. Tsuruta, Molecular dynamics study on evaporation coefficient of biodiesel fuel, Seventh International Conference on Flow Dynamics (2010) 386.

99. B.-Y. Cao, J.-F. Xie, S. S. Sazhin, Molecular dynamics study on evaporation and condensation of n-dodecane at liquid-vapor phase equilibria, AIP The Journal of Chemical Physics 134 (16) (2011) 164309

100. J.-F. Xie, S. S. Sazhin, B.-Y. Cao, Molecular dynamics study of the processes in the vicinity of the n-dodecane vapour/liquid interface, Physics of Fluids 23 (11) (2011) 112104. 